\title{
Mesures invariantes pour les fractions rationnelles géométriquement finies
}

\author{
par
}

\author{
Guillaume Havar d (Orléans)
}

\begin{abstract}
Let $T$ be a geometrically finite rational map, $p(T)$ its petal number and $\delta$ the Hausdorff dimension of its Julia set. We give a construction of the $\sigma$-finite and $T$-invariant measure equivalent to the $\delta$-conformal measure. We prove that this measure is finite if and only if $\frac{p(T)+1}{p(T)} \delta>2$. Under this assumption and if $T$ is parabolic, we prove that the only equilibrium states are convex combinations of the $T$-invariant probability and $\delta$-masses at parabolic cycles.
\end{abstract}

\section{Introduction}

1.1. Généralités. Soit $T$ une fraction rationnelle de degré supérieur ou égal à 2 . On définit l'ensemble de Julia de $T$ comme l'adhérence de l'ensemble des points périodiques répulsifs. On le note $J(T)$ ou plus simplement $J$ et sa dimension de Hausdorff est notée $\delta$.

L'ensemble critique $\mathcal{C}$ de $T$ est l'ensemble fini des points critiques de $T$. On note

$$
\mathcal{P C}=\overline{\bigcup_{n \geq 0} T^{n}(\mathcal{C})}
$$

Dans ce travail, nous nous intéressons à une classe particulière de fractions rationnelles. Il s'agit des fractions rationnelles géométriquement finies.

DÉFInition. Une fraction rationnelle $T$ est dite géométriquement finie si les points de $\mathcal{C} \cap J$ sont pré-périodiques.

La présence de disque de Siegel ou d'anneau de Herman est exclue du cadre géométriquement fini. Par contre, les fractions rationnelles hyperboliques, sous-hyperboliques et paraboliques sont géométriquement finies.

Pour mieux comprendre la géométrie des ensembles de Julia, on étudie un certain nombre de mesures.

1991 Mathematics Subject Classification: 58F23, 28A80, 58F11. 
On pense en premier lieu à la $\delta$-mesure de Hausdorff de $J, \mathcal{H}_{\delta}$. Celle-ci est bien adaptée à l'étude des ensembles de Cantor auto-similaires. Mais l'autosimilarité dans le cas des ensembles de Julia est bien moins "contrôlable" et le calcul, même approché, de la dimension de Hausdorff est un problème délicat (cf. [Bo,Zi], $\left[\mathrm{McMu}^{2}\right]$ ). Il est même difficile de savoir si la masse totale de $\mathcal{H}_{\delta}$ est non nulle, finie ou infinie [Ur $\left.{ }^{1}\right]$. Néanmoins, lorsqu'elle est finie et non nulle, on remarque que pour tout ensemble $A \mathcal{H}_{\delta}$-mesurable et tel que $T$ soit injective sur $A$ on a

$$
\mathcal{H}_{\delta}(T(A))=\int_{A}\left|T^{\prime}\right|^{\delta} d \mathcal{H}_{\delta} .
$$

Cette égalité désigne $\mathcal{H}_{\delta}$ comme une mesure $\delta$-conforme.

DÉfinition. Une mesure $\nu$ est $t$-conforme avec $t>0$ si, pour chaque $A$ sur lequel $T$ est injective, on a

$$
\nu(T(A))=\int_{A}\left|T^{\prime}\right|^{t} d \nu
$$

Dans le cas des fractions rationnelles sans point critique récurrent Urbański a prouvé dans $\left[\mathrm{Ur}^{1}\right]$ qu'il existe toujours une unique probabilité $\delta$-conforme (cf. $\left[\mathrm{McMu}^{1}\right]$ pour le cas moins général des fractions rationnelles géométriquement finies). Cette mesure est sans atome.

Remarque. Dans son article $\left[\mathrm{McMu}^{1}\right]$, McMullen appelle mesures $T$ invariantes de dimension $t$ les mesures $t$-conformes. En ce qui nous concerne, une mesure $\mu$ sera dite $T$-invariante si $\mu\left(T^{-1}(A)\right)=\mu(A)$.

Dans toute la suite nous noterons m l'unique probabilité $\delta$-conforme.

1.2. Enoncé des résultats. Nous étudions l'existence de mesures $T$-invariantes et absolument continues par rapport à $\mathrm{m}$. L'existence d'une telle mesure rend compte d'une certaine "régularité" du système dynamique. On sait par exemple que l'hyperbolicité assure l'existence d'une unique probabilité $T$-invariante, ergodique (cf. $[\mathrm{Su}],\left[\mathrm{Ru}^{2}\right]$ ). De même, dans le cas sous-hyperbolique une telle probabilité existe (cf. $\left[\mathrm{De}, \mathrm{Ur}^{1}\right]$ ).

Le cas parabolique est plus délicat. Une mesure $T$-invariante et équivalente à $\mathrm{m}$ existe. Mais elle n'est finie que si et seulement si une certaine condition faisant intervenir $\delta$ et le nombre de pétales de $T$ est vérifiée (cf. $[\mathrm{Aa}, \mathrm{De}, \mathrm{Ur}])$.

Dans le cas géométriquement fini dans toute sa généralité, les résultats sont du même type que ceux obtenus dans le cas parabolique. Nous prouvons

THÉORÈme 1.1. Soit $T$ une fraction rationnelle géométriquement finie de degré supérieur ou égal à 2 . Il existe une unique mesure $\mu T$-invariante, $\sigma$-finie et équivalente à $\mathrm{m}$. 
L'unicité de la mesure doit être comprise ici comme unicité à une constante multiplicative près.

La rédaction de ce papier à peine achevée, nous avons appris la parution de l'article [ $\left.\mathrm{Ur}^{2}\right]$ de Urbański qui rend caduc ce résultat. Dans cet article Urbański traite la situation beaucoup plus générale des fractions rationnelles sans point critique récurrent. Il prouve que dans ce cas il existe toujours une mesure $T$-invariante, $\sigma$-finie et équivalente à l'unique mesure $\delta$-conforme dont il a prouvé l'existence dans [ $\left.\mathrm{Ur}^{1}\right]$. Néanmoins, pour prouver 1.2 et 1.3 nous aurons besoin de la construction de la mesure $T$-invariante que nous avions utilisée. Elle figure donc dans la troisième section.

Si $p(T)$ est le nombre de pétales de $T$, nombre introduit par McMullen dans $\left[\mathrm{McMu}^{1}\right]$, on a

THÉORÈME 1.2. La mesure T-invariante et équivalente à $\mathrm{m}$ est finie si et seulement si

$$
\alpha(T)=\frac{p(T)+1}{p(T)} \delta>2 .
$$

Lorsque $p(T)$ vaut 0 , on pose $\alpha(T)=\infty$ et le théorème s'applique $(p(T)=0$ correspond aux cas hyperbolique et sous-hyperbolique).

En ce qui concerne le cas particulier des fractions rationnelles paraboliques, nous donnons une autre démonstration des théorèmes 1.1 et 1.2 que celle exposée dans $[\mathrm{Aa}, \mathrm{De}, \mathrm{Ur}]$.

Les démonstrations que nous ferons n'utiliserons que des résultats élémentaires de théorie ergodique, le théorème de distorsion de Kobe et le théorème de la fleur de Leau-Fatou.

Enfin, dans le dernier paragraphe, nous supposerons que la fraction rationnelle est parabolique et $\alpha(T)$ strictement supérieur à 2. Nous noterons $\mu$ l'unique probabilité $T$-invariante et équivalente à $\mathrm{m}$ et nous noterons $\Omega$ l'ensemble des points paraboliques. Nous montrerons alors le

THÉORÈme 1.3. Soit $T$ est une fraction rationnelle parabolique telle que $\alpha(T)>2$. Si $\nu$ est un état d'équilibre associé à $T$, alors

$$
\nu=\beta \mu+\sum_{\omega \in \Omega} \beta_{\omega} \delta_{\omega}
$$

où, pour chaque $\omega, \delta_{\omega}$ désigne la masse de Dirac en $\omega$ et où $\beta$ et $\left(\beta_{\omega}\right)_{\omega \in \Omega}$ sont des réels positifs de somme totale égale à 1 .

Remarque. Dans ce théorème, si $\omega_{1}$ et $\omega_{2}$ sont sur le même cycle parabolique, on a $\beta_{\omega_{1}}=\beta_{\omega_{2}}$.

Pour la définition de la notion d'état d'équilibre, nous renvoyons à la section suivante. 


\subsection{Quelques rappels}

1.3.1. Dynamique holomorphe. Si $\omega$ est sur un cycle parabolique de longueur $k$ et de multiplicateur égal à $\exp (2 i \pi p / q)$, alors $\omega$ est un point fixe parabolique de multiplicateur égal à 1 pour $T^{\circ q k}$ et, dans un de ses voisinages $\mathcal{V}$, on a

$$
\forall z \in \mathcal{V} \quad T^{\circ q k}(z)=z+a_{\omega}(z-\omega)^{p(\omega)+1}+\mathcal{O}\left((z-\omega)^{p(\omega)+2}\right) .
$$

L'entier $p(\omega)$ est supérieur ou égal à 1 . C'est le nombre de pétales attractifs de la fleur de Leau-Fatou associée à $\omega$ pour $T$ [Mi]. On vérifie que le nombre de pétales associé à $\omega$ pour $T$ est le même que celui associé à n'importe quel autre point du cycle parabolique pour n'importe laquelle des itérées de $T$.

Si maintenant $c$ est un point de $\mathcal{P C}$ pré-périodique dont la $q^{\text {ième }}$ itérée est dans un cycle parabolique, on dit alors que $c$ est pré-parabolique. Le point $T^{\circ q}(c)$ est sur un cycle parabolique, on note $p\left(T^{\circ q}(c)\right)$ son nombre de pétales. Dans un voisinage $\mathcal{V}_{c}$ de $c$, on a

$$
\forall z \in \mathcal{V}_{c} \quad T^{\circ q}(z)=T^{\circ q}(c)+a_{c}(z-c)^{d_{c}}+\mathcal{O}\left((z-c)^{d_{c}+1}\right) .
$$

On définit, suivant McMullen $\left[\mathrm{McMu}^{1}\right]$, le nombre de pétales de $c, p(c)$, par

$$
p(c)=d_{c} p\left(T^{\circ q}(c)\right) .
$$

Le nombre de pétales de $T, p(T)$, est alors défini comme étant le maximum des nombres de pétales des points paraboliques et pré-paraboliques.

La dénomination de nombre de pétales pour les points critiques préparaboliques s'explique de la manière suivante. On construit dans un voisinage de $c$ une fonction holomorphe $g_{c}$ solution de $T^{\circ q} \circ g_{c}=T^{\circ k} \circ T^{\circ q}$, où $k$ est un entier tel que $T^{\circ q}(c)$ est un point fixe parabolique de $T^{\circ k}$. On vérifie que $c$ est un point fixe parabolique de $g_{c}$ et que, dans un voisinage $\mathcal{V}_{c}$ de $c$, on a

$$
\forall z \in \mathcal{V}_{c} \quad g_{c}(z)=z+b_{c}(z-c)^{p(c)+1}+\mathcal{O}\left((z-c)^{p(c)+2}\right) .
$$

De plus, l'équation $T^{\circ q} \circ g_{c}=T^{\circ k} \circ T^{\circ q}$ nous assure que $g_{c}$ respecte la $\delta$-conformité de $\mathrm{m}:$ si $A \subset \mathcal{V}_{c}$ est m-mesurable alors

$$
\mathrm{m}\left(g_{c}(A)\right)=\int_{A}\left|g_{c}^{\prime}\right|^{\delta} d \mathrm{~m} .
$$

Pour en terminer avec ces rappels, nous donnons une proposition qui rend compte de la dynamique au voisinage des points fixes paraboliques de multiplicateur égal à 1 et au voisinage des points pré-paraboliques. Soit $\omega$ un tel point. Si $\omega$ est un point fixe parabolique, on pose $f_{\omega}=T$, et si $\omega$ est un point pré-parabolique, on pose $f_{\omega}=g_{\omega}$.

Proposition 1.4. Il existe un voisinage $\mathcal{V}_{\omega}$ de $\omega$ tel que :

- il existe une unique branche holomorphe de $f_{\omega}^{-1}$ définie sur $\mathcal{V}_{\omega}$ et fixant $\omega$, 
- $f_{\omega}^{-1}\left(\mathcal{V}_{\omega} \cap J\right) \subset \mathcal{V}_{\omega} \cap J$

- il existe une constante $\lambda>1$ telle que, pour tout $z \in J \cap\left(\mathcal{V}_{\omega} \backslash f_{\omega}^{-1}\left(\mathcal{V}_{\omega}\right)\right)$ et tout $n \in \mathbb{N}$, on ait

$$
\frac{1}{\lambda} n^{(p(\omega)+1) / p(\omega)} \leq\left|\left(f_{\omega}^{\circ n}\right)^{\prime}\left(f_{\omega}^{-n}(z)\right)\right| \leq \lambda n^{(p(\omega)+1) / p(\omega)} .
$$

Pour une preuve voir $[\mathrm{Aa}, \mathrm{De}, \mathrm{Ur}]$.

1.3.2. Pression, transition de phase et états d'équilibre. Soient $X$ un espace métrique compact et $S$ une transformation continue de $X$ dans luimême. On note $M_{S}(X)$ l'ensemble des mesures de probabilité $S$-invariantes supportées par $X$. A toute mesure $S$-invariante, $\mu$, on associe son entropie $h_{\mu(S)}$; pour une définition de l'entropie d'une mesure on pourra se reporter à $\left[\mathrm{Wa}^{2}\right]$.

Si $\varphi$ est une fonction continue sur $X$ et à valeurs réelles, le principe variationnel (cf. $\left[\right.$ Bow], $\left[\mathrm{Ru}^{1}\right]$ et aussi $\left[\mathrm{Wa}^{1}\right]$ ) permet de définir la pression, $p(\varphi, S)$, de $\varphi$ de la manière suivante :

$$
p(\varphi, S)=\sup \left\{h_{\mu(S)}+\int_{X} \varphi d \mu: \mu \in M_{S}(X)\right\} .
$$

Les mesures qui réalisent ce supremum sont appelées $\varphi$-états d'équilibre. Lorsque plusieurs états d'équilibre cohabitent on parle de transition de phase.

Nous prendrons comme compact l'ensemble de Julia $J$ et comme transformation la fraction rationnelle $T$. Lorsque $T$ n'a pas de point critique dans $J$ on définit une fonction $p$ comme suit :

$$
\forall t \in \mathbb{R} \quad p(t)=p\left(-t \log \left|T^{\prime}\right|, S\right) .
$$

Dans le cas hyperbolique, $p$ est une fonction réelle-analytique et strictement décroissante sur $\mathbb{R}$. Son unique zéro est la dimension de Hausdorff, $\delta$, de $J$.

Dans le cas parabolique (cf. [De, $\left.\mathrm{Ur}^{2}\right]$ ), $p$ est continue, décroissante et positive :

- $p(t)>0$ si $t<\delta$.

- $p(t)=0$ si $t \geq \delta$.

Pour une étude détaillée de $p$ lorsque $T$ est un polynôme nous renvoyons à [Sm]. Généralement, la régularité de $p$ en un point $t$ et l'unicité du $t$-état d'équilibre associé vont de pair. Le changement de comportement de $p$ au point $\delta$ correspond à une transition de phase. Dans le cas parabolique, on a, en $t=\delta$, cohabitation de plusieurs états d'équilibre. L'objet du théorème 1.3 est de donner une description complète de cette transition de phase.

1.3.3. Plan général de l'étude. On peut, sans changer la nature de notre problème, remplacer $T$ par une de ses itérées pour supposer d'une part que 
les cycles paraboliques sont d'ordre 1 et de multiplicateur égal à 1 et d'autre part que les points critiques ont leur image par cette itérée qui est un point fixe.

Si $\mu_{k}$ est une mesure $T^{\circ k_{-}}$invariante, équivalente à $\mathrm{m}$ et $\sigma$-finie, en posant

$$
\mu=\mu_{k}+T^{*} \mu_{k}+\ldots+\left(T^{\circ(k-1)}\right)^{*} \mu_{k},
$$

on définit une mesure $T$-invariante, équivalente à $\mathrm{m}$ et $\sigma$-finie. De plus, $\mu$ est finie si et seulement si $\mu_{k}$ l'est. Cette remarque nous autorise à travailler avec n'importe laquelle des itérées de $T$ et désormais $T$ désignera en fait $T^{\circ k}$, avec $k$ tel que nous le désirons.

Le résultat étant connu pour les ensembles de Julia sous-hyperboliques, on peut supposer que $T$ possède au moins un point périodique parabolique ou attractif.

Dans le cas géométriquement fini on peut donc supposer que $J$ est compact dans $\mathbb{C}$ et que $\infty$ est un point critique périodique ou d'orbite infinie.

Dans la première partie nous étudions la mesure $\delta$-conforme. Nous donnons des estimations de la m-mesure de certains ensembles dans le voisinage des points de $\mathcal{P C} \cap J$. Nous rappelons aussi quelques propriétés de m, notamment une formule de changement de variables faisant intervenir l'opérateur de transfert.

Dans la seconde partie nous donnons une construction de la mesure $T$ invariante. Nous utilisons cette construction pour démontrer le théorème 1.2 et pour obtenir des estimations de la $\mu$-mesure d'ensembles au voisinage des points de $\mathcal{P C} \cap J$. En particulier, suivant la terminologie de Urbański dans $\left[\mathrm{Ur}^{2}\right]$, nous verrons que l'ensemble des points d'infinie condensation est inclus dans l'ensemble des points paraboliques.

Dans la dernière partie nous supposons $\alpha(T)>2$. Nous étudions la densité de $\mu$ par rapport à $\mathrm{m}$. Puis nous démontrons le théorème 1.3.

\section{Etude de la mesure $\delta$-conforme}

2.1. Premières estimations. Sous l'hypothèse $T$ géométriquement finie, $\mathcal{P C} \cap J$ est un ensemble fini. Il est composé des points fixes paraboliques, des points de $\mathcal{C} \cap J$ et de leurs images par $T$.

Soit $\omega$ un élément de $\mathcal{P C} \cap J$. Comme nous l'avons fait en introduction, on lui associe $f_{\omega}$, une fonction définie dans un voisinage, $\mathcal{M}_{\omega}$, de $\omega$. On a $f_{\omega}=T$ si $\omega$ est un point fixe de $T$ et $f_{\omega}$ est une solution de $T \circ T^{\circ k}=T^{\circ k} \circ f$ si $\omega$ est un point de $\mathcal{P C}$ prépériodique. Dans les deux cas, on vérifie que $\omega$ est un point fixe de $f_{\omega}$ et pour tout $z \in \mathcal{M}_{\omega}$, on a

$$
f_{\omega}(z)=\left\{\begin{array}{l}
\omega+\lambda_{\omega}(z-\omega)+\mathcal{O}\left((z-\omega)^{2}\right),\left|\lambda_{\omega}\right|>1, \quad \text { si } \omega \text { est répulsif } \\
z+a_{\omega}(z-\omega)^{p(\omega)+1}+\mathcal{O}\left((z-\omega)^{p(\omega)+2}\right), \\
a_{\omega} \neq 0,
\end{array}\right.
$$


On note alors $\mathcal{M}_{\omega, n}$ l'ensemble des points de $\mathcal{M}_{\omega} \cap J$ qui quittent $\mathcal{M}_{\omega}$ après $n$ itérées de $f_{\omega}$. On remarque que

$$
\mathcal{M}_{\omega, n}=f_{\omega}^{-(n-1)}\left(\mathcal{M}_{\omega} \backslash f_{\omega}^{-1}\left(\mathcal{M}_{\omega}\right) \cap J\right) .
$$

Comme $f_{\omega}$ respecte la $\delta$-conformité de $\mathrm{m}$, on en déduit

$$
\mathrm{m}\left(\mathcal{M}_{\omega} \backslash f_{\omega}^{-1}\left(\mathcal{M}_{\omega}\right)\right)=\int_{\mathcal{M}_{\omega, n}}\left|\left(T^{\circ(n-1)}\right)^{\prime}\right|^{\delta} d \mathrm{~m} .
$$

Tout ceci nous conduit à énoncer la

Proposition 2.1. Il existe une constante $\lambda>1$ telle que pour tout $n \geq 1$ on ait :

- si $\omega$ est un point fixe répulsif de $f_{\omega}$ alors

$$
\frac{1}{\lambda^{n \delta}} \leq \mathrm{m}\left(\mathcal{M}_{\omega, n}\right) \leq \frac{1}{\lambda^{(n-1) \delta}},
$$

- si $\omega$ est un point fixe parabolique de $f_{\omega}$ alors

$$
\frac{1}{\lambda n^{\alpha(\omega)}} \leq \mathrm{m}\left(\mathcal{M}_{\omega, n}\right) \leq \frac{\lambda}{n^{\alpha(\omega)}} .
$$

2.2. Une formule de changement de variables. La formule que nous rappelons fait intervenir l'opérateur de transfert $\mathcal{L}$. Pour toute fonction $\mathrm{m}-$ mesurable $f$, on définit $\mathcal{L}(f)$ m-presque partout par

$$
\mathcal{L}(f)(x)=\sum_{T(y)=x}\left|T^{\prime}(y)\right|^{-\delta} f(y) .
$$

En itérant cette formule on obtient, pour tout $k \in \mathbb{N}$,

$$
\mathcal{L}^{\circ k}(f)(x)=\sum_{T^{\circ k}(y)=x}\left|\left(T^{\circ k}\right)^{\prime}(y)\right|^{-\delta} f(y) .
$$

La formule de changement de variables est alors, pour tout ensemble $A$ m-mesurable et toute fonction $f$ élément de $L^{1}(\mathrm{~m})$,

$$
\forall k \in \mathbb{N} \quad \int_{T^{-k}(A)} f d \mathrm{~m}=\int_{A} \mathcal{L}^{\circ k}(f) d \mathrm{~m} .
$$

2.3. Nouvelles estimations. On donne la version suivante du théorème de distorsion bornée de Kobe (cf. [Po]).

Proposition 2.2. Soient $\mathcal{V} \subset \overline{\mathcal{V}} \subset \mathcal{V}^{\prime}$ deux domaines simplement connexes bornés de $\widehat{\mathbb{C}}$. Pour tout $M>0$ il existe une fonction $\varepsilon: \mathbb{R}^{+} \rightarrow \mathbb{R}^{+}$ bornée et tendant vers 0 en 0 telle que pour toute fonction $f$ holomorphe injective de $\mathcal{V}^{\prime}$ dans $\{|z|<M\}$ on ait

$$
\forall(x, y) \in \mathcal{V} \times \mathcal{V} \quad e^{-\varepsilon(|x-y|)} \leq \frac{\left|f^{\prime}(x)\right|}{\left|f^{\prime}(y)\right|} \leq e^{\varepsilon(|x-y|)}
$$


Nous allons appliquer ce résultat aux itérées successives de $T$ et nous ne pourrons le faire qu'à condition d'être certain que ces itérées sont univalentes. Nous ne pourrons donc l'appliquer qu'à condition d'être éloigné de $\mathcal{P C}$. Nous prouvons

Proposition 2.3. (a) Si $\mathcal{B}$ et $\mathcal{B}^{\prime}$ sont deux boules éloignées de $\mathcal{P C}$ et si la $\mathrm{m}$-mesure de $\mathcal{B}$ est non nulle alors pour tout entier $N$,

$$
\sum_{n=0}^{N} \mathrm{~m}\left(T^{-n}\left(\mathcal{B}^{\prime}\right)\right) \sim \frac{\mathrm{m}\left(\mathcal{B}^{\prime}\right)}{\mathrm{m}(\mathcal{B})} \sum_{n=0}^{N} \mathrm{~m}\left(T^{-n}(\mathcal{B})\right) .
$$

(b) Si $\mathcal{V}$ et $\mathcal{V}^{\prime}$ sont deux ouverts simplement connexes, éloignés de $\mathcal{P C}$ et vérifiant les hypothèses permettant d'appliquer la proposition 2.2 , il existe alors une constante $\lambda>1$ telle que, quelle que soit $\mathcal{V}_{k}$, une composante connexe de $T^{-k}(\mathcal{V})$, on ait

$$
\forall n \in \mathbb{N} \forall\left(x, x^{\prime}\right) \in \mathcal{V}_{k} \times \mathcal{V}_{k} \quad \frac{1}{\lambda} \leq \frac{\mathcal{L}^{\circ n}(1)(x)}{\mathcal{L}^{\circ n}(1)\left(x^{\prime}\right)} \leq \lambda .
$$

Démonstration. (a) Les points d'accumulation de $\mathcal{P C}$ sont soit dans $J$, des points fixes paraboliques ou répulsifs, soit en dehors de $J$, des points fixes attractifs ou super-attractifs. Ils sont donc en nombre fini. Cela implique qu'il est possible d'isoler $\mathcal{P C}$ dans une réunion finie de boules disjointes deux à deux.

Si maintenant $\mathcal{B}$ et $\mathcal{B}^{\prime}$ sont deux boules éloignées de $\mathcal{P C}$, la remarque ci-dessus nous indique que l'on peut construire deux ouverts simplement connexes $\mathcal{V}$ et $\mathcal{V}^{\prime}$ éloignés de $\mathcal{P C}$, contenant $\mathcal{B} \cup \mathcal{B}^{\prime}$ et vérifiant les hypothèses nous permettant d'appliquer la proposition 2.2. Le fait que $\mathcal{V}^{\prime}$ soit éloigné de $\mathcal{P C}$ nous assure que chaque branche holomorphe de $T^{-n}$ définie sur $\mathcal{V}^{\prime}$ réalise un difféomorphisme sur son image. On peut donc leur appliquer la proposition 2.2 et il existe une constante $\lambda \geq 1$ telle que pour toute branche holomorphe de $T^{-n}$ définie sur $\mathcal{V}^{\prime}$ et tout $(x, y) \in \mathcal{V} \times \mathcal{V}$, on ait

$$
\frac{1}{\lambda} \leq \frac{\left|\left(T^{-n}\right)^{\prime}(x)\right|}{\left|\left(T^{-n}\right)^{\prime}(y)\right|} \leq \lambda .
$$

La constante $\lambda$ ne dépendant ni de $n$ ni de la branche de $T^{-n}$ que l'on considère, on en déduit que, pour tout entier $k$ et tout $(x, y) \in \mathcal{V} \times \mathcal{V}$, on a

$$
\frac{1}{\lambda} \mathcal{L}^{\circ k}(1)(y) \leq \mathcal{L}^{\circ k}(1)(x) \leq \lambda \mathcal{L}^{\circ k}(1)(y) .
$$

On vient donc de montrer que pour toute boule $\mathcal{B}^{\prime}$ éloignée de $\mathcal{P C}$ on a

$$
\forall x \in \mathcal{B}^{\prime} \quad \mathrm{m}\left(\mathcal{B}^{\prime}\right) \mathcal{L}^{\circ k}(1)(x) \sim \int_{\mathcal{B}^{\prime}} \mathcal{L}^{\circ k}(1) d \mathrm{~m} .
$$


Or, la formule de changement de variables nous indique que

$$
\int_{\mathcal{B}^{\prime}} \mathcal{L}^{\circ k}(1) d \mathrm{~m}=\mathrm{m}\left(T^{-k}\left(\mathcal{B}^{\prime}\right)\right) .
$$

Comme $\mathrm{m}(\mathcal{B})>0$, l'égalité ci-dessus et $(2.2)$ nous donnent

$$
\sum_{n=0}^{N} \mathrm{~m}\left(T^{-n}\left(\mathcal{B}^{\prime}\right)\right) \sim \frac{\mathrm{m}\left(\mathcal{B}^{\prime}\right)}{\mathrm{m}(\mathcal{B})} \sum_{n=0}^{N} \mathrm{~m}\left(T^{-n}(\mathcal{B})\right) .
$$

(b) Comme $\mathcal{V}^{\prime}$ est éloigné de $\mathcal{P C}$, la proposition 2.2 nous indique l'existence d'une constante $\lambda>1$, indépendante de $n$ et de la branche holomorphe de $T^{-n}$ que l'on considère, telle que

$$
\forall\left(z, z^{\prime}\right) \in \mathcal{V} \times \mathcal{V} \quad \frac{1}{\lambda} \leq \frac{\left|\left(T^{-n}\right)^{\prime}(z)\right|}{\left|\left(T^{-n}\right)^{\prime}\left(z^{\prime}\right)\right|} \leq \lambda .
$$

Soient $x$ et $x^{\prime}$ deux éléments d'une même composante connexe, $\mathcal{V}_{k}$, de $T^{-k}(\mathcal{V})$. Soient $y$ et $y^{\prime}$ deux antécédents respectivement de $x$ et de $x^{\prime}$ qui appartiennent à une même composante connexe, $\mathcal{V}_{n+k}$, de $T^{-(n+k)}(\mathcal{V})$. On pose $z=T^{k}(x)$ et $z^{\prime}=T^{k}\left(x^{\prime}\right)$. On a

$$
\left(T^{\circ n}\right)^{\prime}(y)=\left(T^{n+k}\right)^{\prime}(y) \cdot\left(T^{k}\right)^{\prime}(x)
$$

et aussi

$$
\left(T^{o n}\right)^{\prime}\left(y^{\prime}\right)=\left(T^{n+k}\right)^{\prime}\left(y^{\prime}\right) \cdot\left(T^{k}\right)^{\prime}\left(x^{\prime}\right) .
$$

Si on note $T^{-k}$ (respectivement $T^{-(n+k)}$ ) la branche holomorphe de $T^{-k}$ (respectivement de $T^{-(n+k)}$ ) qui envoie $\mathcal{V}$ sur $\mathcal{V}_{k}$ (respectivement sur $\mathcal{V}_{n+k}$ ), on a alors

et aussi

$$
\left(T^{\circ n}\right)^{\prime}(y)=\frac{1}{\left(T^{-(n+k)}\right)^{\prime}(z) \cdot\left(T^{-k}\right)^{\prime}(z)}
$$

$$
\left(T^{\circ n}\right)^{\prime}\left(y^{\prime}\right)=\frac{1}{\left(T^{-(n+k)}\right)^{\prime}\left(z^{\prime}\right) \cdot\left(T^{-k}\right)^{\prime}\left(z^{\prime}\right)} .
$$

De (2.4) on déduit alors l'existence d'une constante $\lambda>1$ telle que

$$
\frac{1}{\lambda} \leq \frac{\left|\left(T^{\circ n}\right)^{\prime}(y)\right|}{\left|\left(T^{\circ n}\right)^{\prime}\left(y^{\prime}\right)\right|} \leq \lambda .
$$

$\lambda$ ne dépendant que de $\mathcal{V}$ et de $\mathcal{V}^{\prime}$, en sommant sur tous les antécédents de $x$ et de $x^{\prime}$ on obtient bien

$$
\forall n \in \mathbb{N} \quad \frac{1}{\lambda} \leq \frac{\mathcal{L}^{\circ n}(1)(x)}{\mathcal{L}^{\circ n}(1)\left(x^{\prime}\right)} \leq \lambda .
$$

Remarque. Cette proposition nous donne une démonstration d'un résultat énoncé dans $\left[\mathrm{McMu}^{1}\right]$. En effet, dans le cas géométriquement fini, on montre que la série de Poincaré diverge sur $\widehat{\mathbb{C}} \backslash \mathcal{P C}$. 
Si $x$ est un élément de $\widehat{\mathbb{C}} \backslash \mathcal{P C}$, la série de Poincaré en $x$ est définie par

$$
S P(x)=\sum_{k=0}^{\infty} \mathcal{L}^{\circ k}(1)(x) .
$$

Comme $x$ est éloigné de $\mathcal{P C}$, on peut trouver une boule $\mathcal{B}^{\prime}$ éloignée de $\mathcal{P C}$ qui contient $x$. Soit une seconde boule éloignée de $\mathcal{P C}, \mathcal{B}$, telle que $\mathrm{m}(\mathcal{B})>0$. Comme $(J, T, \mathrm{~m})$ est conservatif $\left(\mathrm{cf}\right.$. $\left.\left[\mathrm{Ur}^{2}\right]\right)$, on a

$$
\sum_{k=0}^{\infty} \mathrm{m}\left(T^{-k}(\mathcal{B})\right)=\infty
$$

L'estimation (2.3) nous donne alors la divergence de la série de Poincaré sur $\mathcal{B}$. On conclut ensuite en utilisant (2.2).

3. Etude de la mesure $T$-invariante. On rappelle que Urbański a prouvé, dans $\left[\mathrm{Ur}^{2}\right]$, qu'il existait pour toute fraction rationnelle sans point critique récurrent une unique mesure $\sigma$-finie, $T$-invariante et équivalente à l'unique mesure $\delta$-conforme. Dans le cas particulier de fraction rationnelle sans point critique récurrent auquel nous nous intéressons, nous donnons une construction de cette mesure qui nous servira pour obtenir un certain nombre d'estimations.

3.1. Une construction de $\mu$. On commence par construire un ouvert $\mathcal{U}$ éloigné de $\mathcal{P C}$. Dans la suite nous aurons besoin de connaître le comportement des itérées des points de cet ouvert. Autour de chaque point fixe, $\omega$, de $\mathcal{P C} \cap J$ on considère une boule de rayon très petit, $\mathcal{M}_{\omega}$. Si $\omega$ admet un antécédent critique $c$ on note $\mathcal{M}_{c}$ la composante connexe de $T^{-1}\left(\mathcal{M}_{\omega}\right)$ qui contient $c$. On choisit les rayons des boules $\mathcal{M}_{\omega}$ suffisamment petits pour que tous ces ensembles soient disjoints deux à deux. On pose alors

$$
\mathcal{M}=\bigcup_{\omega \in \mathcal{P} \mathcal{C} \cap J} \mathcal{M}_{\omega} \quad \text { et } \quad \mathcal{U}=J \backslash \mathcal{M} .
$$

On utilise maintenant une procédure classique en théorie ergodique pour constuire une mesure invariante (cf. $[\mathrm{Fo}])$. Comme $(J, T, \mathrm{~m})$ est conservatif, tout ensemble de m-mesure positive est récurrent. A tout ensemble $A$ de m-mesure non nulle on associe alors $N_{A}$ l'application définie pour m-presque tout $x$ par

$$
N_{A}(x)=\inf \left\{n \geq 1: T^{\circ n}(x) \in A\right\} .
$$

La transformation induite est alors définie par $T_{A}(x)=T^{\circ N_{A}}(x)$.

A l'aide de limites de Banach nous allons construire une mesure $\nu_{\mathcal{U}}$ à support inclus dans $\mathcal{U}$ et $T_{\mathcal{U}}$-invariante. Le fait d'avoir pris $\mathcal{U}$ éloigné de $\mathcal{P C}$ va nous assurer que cette mesure est équivalente à $\mathrm{m}$. 
Définition. Une limite de Banach $\Lambda$ est une forme linéaire bornée, définie sur l'ensemble des suites bornées à valeurs réelles et telle que

(i) $\Lambda(1)=1$,

(ii) $\|\Lambda\|=1$,

(iii) $\Lambda\left(\left(x_{n}\right)_{n \in \mathbb{N}}\right)=\Lambda\left(\left(x_{n+1}\right)_{n \in \mathbb{N}}\right)$,

(iv) $\liminf _{n \rightarrow \infty} x_{n} \leq \Lambda\left(\left(x_{n}\right)_{n \in \mathbb{N}}\right) \leq \limsup _{n \rightarrow \infty} x_{n}$.

Soit $\Lambda$ une limite de Banach. On définit sur l'ensemble des fonctions continues sur $J$ une forme linéaire bornée en posant pour tout $f$ élément de $\mathcal{C}(J)$

$$
\Psi_{\mathcal{U}}(f)=\Lambda\left[\left(\frac{\sum_{n=0}^{N} \int_{J} \chi_{\mathcal{U}} \circ T^{\circ n} \cdot f \circ T^{\circ n} d \mathrm{~m}}{\sum_{n=0}^{N} \int_{J} \chi_{\mathcal{U}} \circ T^{\circ n} d \mathrm{~m}}\right)_{N \in \mathbb{N}}\right] .
$$

Les propriétés de $\Lambda$ nous assurent que $\Psi_{\mathcal{U}}$ est bien une forme linéaire, positive et bornée. On note $\nu_{\mathcal{U}}$ la mesure positive dont l'existence nous est assurée par le théorème de représentation de Riesz. Pour toute fonction continue $f$ on a

$$
\Psi_{\mathcal{U}}(f)=\int_{J} f d \nu \mathcal{U}
$$

Un calcul fastidieux mais sans difficulté permet de s'assurer que $\nu_{\mathcal{U}}$ est bien une mesure $T_{\mathcal{U}}$-invariante $(\mathrm{cf}$. [Fo]). Il reste à montrer que dans notre situation $\nu_{\mathcal{U}}$ est équivalente à $\mathrm{m}$ restreinte à $\mathcal{U}$. Pour ce faire, on considère une partition finie $\left(\mathcal{U}_{i}\right)_{1 \leq i \leq K}$ de $\mathcal{U}$ telle que chaque $\mathcal{U}_{i}$ soit inclus dans une boule $B_{i}$ éloignée de $\mathcal{P C}$.

Soit $A$ un ensemble m-mesurable inclus dans $\mathcal{U}$. On a

$$
\nu_{\mathcal{U}}(A)=\Lambda\left[\left(\frac{\sum_{n=0}^{N} \mathrm{~m}\left(T^{-n}(A \cap \mathcal{U})\right)}{\sum_{n=0}^{N} \mathrm{~m}\left(T^{-n}(\mathcal{U})\right)}\right)_{N \in \mathbb{N}}\right],
$$

ce qui nous donne aussi

$$
\nu_{\mathcal{U}}(A)=\sum_{i=1}^{K} \Lambda\left[\left(\frac{\sum_{n=0}^{N} \mathrm{~m}\left(T^{-n}\left(A \cap \mathcal{U}_{i}\right)\right)}{\sum_{n=0}^{N} \mathrm{~m}\left(T^{-n}(\mathcal{U})\right)}\right)_{N \in \mathbb{N}}\right] .
$$

Comme les $\mathcal{U}_{i}$ sont inclus dans des boules éloignées de $\mathcal{P C}$, de la proposition 2.3 on déduit l'existence d'une constante $\lambda$ telle que

$$
\forall i \quad \frac{1}{\lambda} \mathrm{m}\left(T^{-n}\left(\mathcal{U}_{i}\right)\right) \mathrm{m}\left(A \cap \mathcal{U}_{i}\right) \leq \mathrm{m}\left(T^{-n}\left(A \cap \mathcal{U}_{i}\right)\right) \leq \lambda \mathrm{m}\left(T^{-n}\left(\mathcal{U}_{i}\right)\right) \mathrm{m}\left(A \cap \mathcal{U}_{i}\right)
$$

et aussi telle que

$$
\forall i \neq j \forall N \quad \frac{1}{\lambda} \leq \frac{\sum_{n=0}^{N} \mathrm{~m}\left(T^{-n}\left(\mathcal{U}_{i}\right)\right)}{\sum_{n=0}^{N} \mathrm{~m}\left(T^{-n}\left(\mathcal{U}_{j}\right)\right)} \leq \lambda .
$$


De ceci on déduit qu'il existe $\lambda$ telle que

$$
\frac{1}{\lambda} \mathrm{m}(A) \leq \nu_{\mathcal{U}}(A) \leq \lambda \mathrm{m}(A) .
$$

Cela implique en particulier que $\nu_{\mathcal{U}}$ est équivalente à m restreinte à $\mathcal{U}$.

A partir de $\nu_{\mathcal{U}}$ on construit la mesure $T$-invariante recherchée en posant, pour tout borélien $A$ de $J$,

$$
\mu(A)=\int_{\mathcal{U}}\left(\sum_{k=0}^{N_{\mathcal{U}}(x)-1} \chi_{A} \circ T^{\circ k}(x)\right) d \nu_{\mathcal{U}}(x) .
$$

Si pour tout entier $n \geq 1$ on note $\mathcal{U}_{n}$ l'ensemble des points $x$ de $J$ tels que $N_{\mathcal{U}}(x)=n$, on a

$$
\mu(A)=\sum_{n=1}^{\infty} \sum_{k=0}^{n-1} \nu_{\mathcal{U}}\left(T^{-k}(A) \cap \mathcal{U}_{n}\right) .
$$

Sous cette forme on vérifie sans problème que $\mu$ est une mesure. La première expression permet quant à elle de vérifier que $\mu$ est $T$-invariante. Pour montrer que $\mu$ est équivalente à $\mathrm{m}$ on effectue le raisonnement suivant.

Soit $A$ un ensemble m-négligeable. Pour tout entier $n, T^{-n}(A)$ est aussi m-négligeable. Comme $\nu_{\mathcal{U}}$ est équivalente à m restreinte à $\mathcal{U}$, on a, pour tout $n, \nu_{\mathcal{U}}\left(T^{-n}(A) \cap \mathcal{U}\right)=0$. On en déduit que $\mu(A)$ vaut 0 et que $\mu$ est absolument continue par rapport à $\mathrm{m}$.

D'autre part, on remarque que, pour tout $A$ inclus dans $J$, on a

$$
\mu(A) \geq \sum_{k=1}^{\infty} \nu_{\mathcal{U}}\left(A \cap \mathcal{U}_{k}\right) .
$$

Soit $A$ de m-mesure non nulle. Comme $(J, T, \mathrm{~m})$ est conservatif, $A$ est mrécurrent, et on a

$$
\mathrm{m}\left(\bigcup_{n=0}^{\infty} T^{-n}(A)\right)=1 .
$$

Il existe donc $l \in \mathbb{N}$ tel que $\mathrm{m}\left(T^{-l}(A) \cap \mathcal{U}\right)$ soit non nulle. On pose $A_{l}=$ $T^{-l}(A) \cap \mathcal{U}$. Comme

$$
\sum_{k=1}^{\infty} \mathrm{m}\left(A_{l} \cap \mathcal{U}_{k}\right)=\mathrm{m}\left(A_{l}\right)>0,
$$

il existe un entier $k$ pour lequel $A_{l} \cap \mathcal{U}_{k}$ est de m-mesure non nulle. $A_{l}$ étant par construction inclus dans $\mathcal{U}$ et $\nu_{\mathcal{U}}$ étant équivalente à m restreinte à $\mathcal{U}$, on en déduit que $\nu_{\mathcal{U}}\left(A_{l} \cap \mathcal{U}_{k}\right)$ est strictement positif. L'inégalité (3.6) permet alors de conclure que $\mu\left(A_{l}\right)$ est strictement positif. Le fait que $A_{l}$ soit inclus dans $T^{-l}(A)$ et la $T$-invariance de $\mu$ nous permettent d'affirmer que $\mu(A)$ est strictement positif. $\mathrm{m}$ est donc absolument continue par rapport à $\mu$. 
Comme $\mathrm{m}$ et $\mu$ sont équivalentes, $(J, T, \mu)$ est un système dynamique conservatif et $\mu$-presque tous les points de $J$ sont inclus dans $\bigcup_{n>1} \mathcal{U}_{n}$. Pour montrer que $\mu$ est $\sigma$-finie, il suffit de vérifier que pour tout $n \geq 1, \mu\left(\mathcal{U}_{n}\right)<\infty$. Or, pour $k<n, T^{-k}\left(\mathcal{U}_{l}\right) \cap \mathcal{U}_{n}$ est l'ensemble vide sauf si $n=k+l$ et dans ce cas $T^{-k}\left(\mathcal{U}_{l}\right) \cap \mathcal{U}_{n}=\mathcal{U}_{n}$. On a donc

$$
\mu\left(\mathcal{U}_{l}\right)=\sum_{n=l}^{\infty} \nu_{\mathcal{U}}\left(\mathcal{U}_{n}\right) \leq \nu_{\mathcal{U}}(\mathcal{U})=1 .
$$

Par suite, la mesure $\mu$ est $\sigma$-finie.

Nous achevons ce paragraphe par la démonstration du théorème 1.1.

Démonstration du théorème 1.1. Il est prouvé dans [ $\left.\mathrm{Ur}^{2}\right]$ que le système dynamique $(J, T, \mathrm{~m})$ est ergodique et conservatif. Cela suffit pour assurer l'unicité, à une constante multiplicative près, de la mesure $T$-invariante, et absolument continue par rapport à $\mathrm{m}$, que l'on vient de construire.

3.2. A quelle condition la mesure T-invariante est-elle finie? En reprenant la construction de $\mu$ on voit que

$$
\mu(J)=\int_{\mathcal{U}} N_{\mathcal{U}} d \nu_{\mathcal{U}},
$$

ce qui peut aussi s'écrire

$$
\mu(J)=\sum_{n=1}^{\infty} n \nu_{\mathcal{U}}\left(\mathcal{U}_{n}\right) .
$$

Ainsi, la mesure $\nu$ est finie si et seulement si la série de terme général $\left(n \nu_{\mathcal{U}}\left(\mathcal{U}_{n} \cap \mathcal{U}\right)\right)_{n \in \mathbb{N}}$ est convergente. Mais on sait, d'après (3.5), que pour tout $A \subset \mathcal{U}$ on a

$$
\frac{1}{\lambda} \leq \frac{\mathrm{m}(A)}{\nu_{\mathcal{U}}(A)} \leq \lambda .
$$

On en déduit que $\mu$ est finie si et seulement si la série de terme général $\left(n \mathrm{~m}\left(\mathcal{U}_{n} \cap \mathcal{U}\right)\right)_{n \in \mathbb{N}}$ est convergente. Pour contrôler la masse totale de $\mu$ il suffit donc d'obtenir une estimation de la m-mesure de l'ensemble des points de $\mathcal{U}$ qui reviennent pour la première fois en $\mathcal{U}$ après $n$ itérées. L'estimation que nous allons obtenir ne concerne pas $\mathrm{m}\left(\mathcal{U}_{n} \cap \mathcal{U}\right)$ mais $\mathrm{m}\left(\mathcal{U}_{n} \cap \mathcal{M}\right)$. L'objet du lemme 3.1 est de montrer le lien qui existe entre $\mathrm{m}\left(\mathcal{U}_{n} \cap \mathcal{U}\right)$ et $\mathrm{m}\left(\mathcal{U}_{n} \cap \mathcal{M}\right)$.

On note $\omega_{0}$ un point fixe parabolique ou un point critique pré-parabolique tel que $\alpha\left(\omega_{0}\right)=\alpha(T)$. Si un point critique $c$ et un point parabolique $\omega$ réalisent cette condition, on pose $\omega_{0}=c$. Cela signifie en particulier que si $\omega_{0}$ est un point fixe parabolique et si $c$ est un point critique pré-parabolique, alors $\alpha\left(\omega_{0}\right)>\alpha(c)$.

On note $\mathcal{M}_{\omega_{0}}$ la composante connexe de $\mathcal{M}$ qui contient $\omega_{0}$. 
LEMME 3.1. Il existe une constante $\lambda>1$ telle que pour tout $n \geq 1$ on ait

$$
\frac{1}{\lambda} \mathrm{m}\left(\mathcal{M}_{\omega_{0}} \cap \mathcal{U}_{n}\right) \leq \mathrm{m}\left(\mathcal{U}_{n+1} \cap \mathcal{U}\right) \leq \lambda \mathrm{m}\left(\mathcal{U}_{n} \cap \mathcal{M}\right) .
$$

Démonstration. On remarque qu'il existe une constante $\lambda>0$ telle que pour tout $A$ inclus dans $\mathcal{U} \cap J$, on ait

$$
\frac{1}{\lambda} \leq \frac{\mathrm{m}(A)}{\mathrm{m}(T(A))} \leq \lambda .
$$

En particulier, on a donc

$$
\frac{1}{\lambda} \mathrm{m}\left(T\left(\mathcal{U}_{n+1} \cap \mathcal{U}\right)\right) \leq \mathrm{m}\left(\mathcal{U}_{n+1} \cap \mathcal{U}\right) \leq \lambda \mathrm{m}\left(T\left(\mathcal{U}_{n+1} \cap \mathcal{U}\right)\right)
$$

Mais $T\left(\mathcal{U}_{n+1} \cap \mathcal{U}\right) \subset \mathcal{U}_{n} \cap \mathcal{M}$. Par conséquent, $\mathrm{m}\left(\mathcal{U}_{n+1} \cap \mathcal{U}\right) \leq \lambda \mathrm{m}\left(\mathcal{U}_{n} \cap \mathcal{M}\right)$.

Pour obtenir l'autre inégalité on raisonne en deux étapes.

D'abord, on suppose que $\omega_{0}$ est un point critique. Alors, $T^{-1}\left(\mathcal{M}_{\omega_{0}}\right)$ est inclus dans $\mathcal{U}$. Cela implique que $\mathcal{U}_{n} \cap \mathcal{M}_{\omega_{0}}$ est inclus dans $T\left(\mathcal{U}_{n+1} \cap \mathcal{U}\right)$ et (3.7) nous donne

$$
\frac{1}{\lambda} \mathrm{m}\left(\mathcal{U}_{n} \cap \mathcal{M}_{\omega_{0}}\right) \leq \mathrm{m}\left(\mathcal{U}_{n+1} \cap \mathcal{U}\right) .
$$

On suppose à présent que $\omega_{0}$ est un point fixe parabolique. Alors $\omega_{0}$ est une racine simple de l'équation $T(z)-\omega_{0}=0$. Soit $\left\{z_{1}, \ldots, z_{p}\right\}$ les autres racines de cette équation comptées sans leur multiplicité. Si l'un des $\left(z_{i}\right)_{1 \leq i \leq p}$ était un point de $\mathcal{P C}$ prépériodique, il serait alors pré-parabolique et l'on aurait $\alpha\left(z_{i}\right) \geq \alpha\left(\omega_{0}\right)$. C'est impossible par hypothèse sur $\omega_{0}$. Donc aucun des antécédents de $\omega_{0}$ n'est critique. Ils sont donc tous dans $\mathcal{U}$, d'où $\mathcal{M}_{\omega_{0}} \subset T(\mathcal{U})$.

On en déduit que $\mathcal{M}_{\omega_{0}} \cap \mathcal{U}_{n} \subset T\left(\mathcal{U} \cap \mathcal{U}_{n+1}\right)$ et la relation (3.7) nous donne alors

$$
\frac{1}{\lambda} \mathrm{m}\left(\mathcal{U}_{n} \cap \mathcal{M}_{\omega_{0}}\right) \leq \mathrm{m}\left(\mathcal{U}_{n+1} \cap \mathcal{U}\right)
$$

Ce lemme et la proposition 2.1 vont nous permettre de démontrer le théorème 1.2 .

Démonstration du théorème 1.2. Le lemme 3.1 nous indique que

$$
\forall n \geq 1 \quad \frac{1}{\lambda} \mathrm{m}\left(\mathcal{M}_{\omega_{0}} \cap \mathcal{U}_{n}\right) \leq \mathrm{m}\left(\mathcal{U}_{n+1} \cap \mathcal{U}\right) \leq \lambda \mathrm{m}\left(\mathcal{U}_{n} \cap \mathcal{M}\right)
$$

Et on sait que $\mu$ est finie si et seulement si la série de terme général $\left(n \mathrm{~m}\left(\mathcal{U}_{n} \cap \mathcal{U}\right)\right)_{n \in \mathbb{N}}$ est convergente.

On remarque à présent que pour tout $\omega$ élément de $\mathcal{P C} \cap J$ on a $\mathcal{M}_{\omega} \cap \mathcal{U}_{n}=$ $\mathcal{M}_{\omega, n}$. Rappelons que $\mathcal{M}_{\omega, n}$ a été introduit au paragraphe 2.1 et désigne l'ensemble des points de $\mathcal{M}_{\omega} \cap J$ qui quittent $\mathcal{M}_{\omega}$ après exactement $n$ itérées de $f_{\omega}$. 
Si $\mathcal{M}_{\omega}$ est une composante connexe de $\mathcal{M}$ qui contient un point fixe parabolique ou un point de $\mathcal{P C}$ pré-parabolique, la proposition 2.1 nous indique que

$$
\mathrm{m}\left(\mathcal{U}_{n} \cap \mathcal{M}_{\omega}\right) \leq \frac{\lambda}{n^{\alpha(T)}} .
$$

En ce qui concerne $\mathcal{M}_{\omega_{0}}$, la composante connexe avec le plus grand nombre de pétales, on a même

$$
\frac{1}{\lambda n^{\alpha(T)}} \leq \mathrm{m}\left(\mathcal{M}_{\omega_{0}} \cap \mathcal{U}_{n}\right) \leq \frac{\lambda}{n^{\alpha(T)}} .
$$

Enfin, pour ce qui concerne les cas répulsifs ou pré-répulsifs, on a

$$
\mathrm{m}\left(\mathcal{U}_{n} \cap \mathcal{M}_{\omega}\right) \leq \frac{1}{\lambda^{n \delta}} .
$$

De toutes ces inégalités on déduit que

$$
\frac{1}{\lambda} \sum_{n=1}^{\infty} \frac{1}{\lambda n^{\alpha(T)-1}} \leq \sum_{n=1}^{\infty} n \mathrm{~m}\left(\mathcal{U}_{n} \cap \mathcal{U}\right) \leq \sum_{n=1}^{\infty}\left(\frac{n}{\lambda^{n \delta}}+\frac{\lambda}{n^{\alpha(T)-1}}\right) .
$$

La série de terme général $\left(n \mathrm{~m}\left(\mathcal{U}_{n} \cap \mathcal{U}\right)\right)_{n \in \mathbb{N}}$ est donc convergente si et seulement si $\alpha(T)>2$. La mesure $\mu$ est donc finie si et seulement si $\alpha(T)>2$.

On termine ce chapitre en donnant des estimations de $\mu\left(\mathcal{M}_{\omega, n}\right)$ en fonction de $n$ et de la nature de $\omega \in \mathcal{P C} \cap J$.

Si $\omega \in \mathcal{P C} \cap J$ est parabolique ou pré-parabolique, on pose $\beta(\omega)=$ $\inf \left\{\alpha(c) \mid c \in \mathcal{C}, T^{\circ k}(c)=\omega\right\}$. Remarquons que l'on a $\beta(\omega) \leq \alpha(\omega)$ avec égalité si et seulement si $\omega$ n'a pas d'autre antécédent danc $\mathcal{P C}$ que lui-même.

Corollaire 3.2. Il existe une constante $\lambda>1$ telle que pour tout élément $\omega$ de $\mathcal{P C} \cap J$ :

- si $\omega$ est répulsif ou pré-répulsif, alors

$$
\forall l \in \mathbb{N} \quad \frac{1}{\lambda^{l+1}} \leq \mu\left(\mathcal{M}_{\omega, l}\right) \leq \frac{1}{\lambda^{l}},
$$

- si $\omega$ est un point fixe parabolique, alors

$$
\forall l \in \mathbb{N} \quad \frac{1}{\lambda} \cdot \frac{1}{l^{\beta(\omega)-1}} \leq \mu\left(\mathcal{M}_{\omega, l}\right) \leq \lambda \frac{1}{l^{\beta(\omega)-1}},
$$

- si $\omega$ est strictement pré-parabolique, alors

$$
\forall l \in \mathbb{N} \quad \frac{1}{\lambda} \cdot \frac{1}{l^{\beta(\omega)}} \leq \mu\left(\mathcal{M}_{\omega, l}\right) \leq \lambda \frac{1}{l^{\beta(\omega)}} .
$$

Démonstration. Soit $\omega$ un élément de $\mathcal{P C} \cap J$ et soient $c_{1}, \ldots, c_{q}$ les points critiques antécédents de $\omega$ tels que $T^{-1}\left(c_{i}\right) \subset \mathcal{U}$. On note $n_{j}=$ $\inf \left\{k \mid T^{\circ k}\left(c_{j}\right)=\omega\right\}$. Par construction de $\mu$ on a

$$
\mu\left(\mathcal{M}_{\omega, l}\right)=\mu\left(\mathcal{U}_{l} \cap \mathcal{M}_{\omega}\right)=\sum_{n=l}^{\infty} \nu_{\mathcal{U}}\left(\mathcal{U}_{n} \cap T^{-(n-l)}\left(\mathcal{M}_{\omega}\right)\right) .
$$


Etant donné (3.5), cela implique

$$
\mu\left(\mathcal{M}_{\omega, l}\right) \sim \sum_{n=l}^{\infty} \mathrm{m}\left(T\left(\mathcal{U} \cap \mathcal{U}_{n} \cap T^{-(n-l)}\left(\mathcal{M}_{\omega}\right)\right)\right) .
$$

Si $\omega$ n'est pas un point fixe de $T$ on a

$$
\sum_{n=l}^{\infty} \mathrm{m}\left(T\left(\mathcal{U} \cap \mathcal{U}_{n} \cap T^{-(n-l)}\left(\mathcal{M}_{\omega}\right)\right)\right)=\sum_{i=1}^{q} \mathrm{~m}\left(\mathcal{M}_{c_{i}, l+n_{i}+1}\right) .
$$

Si $\omega$ est un point fixe de $T$ on a

$$
\sum_{n=l}^{\infty} \mathrm{m}\left(T\left(\mathcal{U} \cap \mathcal{U}_{n} \cap T^{-(n-l)}\left(\mathcal{M}_{\omega}\right)\right)\right)=\sum_{i=1}^{q} \sum_{n=l+n_{i}+1}^{\infty} \mathrm{m}\left(\mathcal{M}_{c_{i}, n}\right) .
$$

Dans les deux cas la proposition 2.1 donne les estimations désirées.

4. Etude des états d'équilibre. Dans cette dernière section nous supposerons que $\alpha(T)$ est strictement plus grand que 2. La mesure $T$-invariante $\mu$ est donc finie.

Le premier paragraphe va être consacré à l'étude de la densité de $\mu$ par rapport à $\mathrm{m}$. Nous obtiendrons un certain nombre d'estimations que nous utiliserons dans le second paragraphe. Dans le dernier paragraphe nous supposerons que la fraction rationnelle $T$ est parabolique. Nous démontrerons le théorème 1.3 qui décrit entièrement les états d'équilibre de $T$.

4.1. Etude de la densité de $\mu$. On rappelle que $\mathcal{L}$ désigne l'opérateur de transfert. Pour tout $x$ dans $J \backslash \mathcal{P C}$ on pose

$$
g(x)=\liminf _{n \rightarrow \infty} \mathcal{L}^{\circ n}(1)(x) .
$$

Nous allons vérifier que $g \mathrm{~m}$ est une mesure finie et $T$-invariante. Comme $g \mathrm{~m}$ est absolument continue par rapport $\mathrm{m}$ on aura alors prouvé que $g$ est, à une constante multiplicative près, la densité de l'unique probabilité invariante et équivalente à $\mathrm{m}$.

Dans un premier temps, on remarque que $g$ est m-intégrable. En effet, la formule de changement de variables (cf. 2.1) nous dit que pour tout $n$ on a

$$
\int_{J} \mathcal{L}^{\circ n}(1) d \mathrm{~m}=\mathrm{m}\left(T^{-n}(J)\right)=1 .
$$

Si on applique le lemme de Fatou à la suite de fonctions positives $\left(\mathcal{L}^{\circ n}(1)\right)_{n \in \mathbb{N}}$ on obtient

$$
\int_{J} g d \mathrm{~m} \leq \liminf _{n \rightarrow \infty} \int_{J} \mathcal{L}^{\circ n}(1) d \mathrm{~m}=1
$$


Comme $g$ est un élément de $L^{1}(\mathrm{~m})$ on peut lui appliquer la formule de changement de variables, qui nous donne

$$
\int_{J} \mathcal{L}(g) d \mathrm{~m}=\int_{J} g d \mathrm{~m} .
$$

Or, par construction, il est clair que $\mathcal{L}(g) \leq g$. On en déduit donc que $\mathcal{L}(g)=$ $g$ m-presque partout. Cette égalité implique, via la formule de changement de variables, que $g \mathrm{~m}$ est une mesure $T$-invariante.

Pour l'instant rien ne nous assure que $g$ n'est pas la fonction nulle mpresque partout et $g \mathrm{~m}$ la mesure nulle. Pour lever cette ambiguïté, on remarque que si $\mathcal{B}$ désigne une boule éloignée de $\mathcal{P C}$ et ayant une intersection non vide avec $J$, alors $\mathrm{m}(\mathcal{B})>0$ et $(2.3)$ nous indique que

$$
\forall x \in \mathcal{B} \quad \mathcal{L}^{\circ n}(1)(x) \sim \int_{\mathcal{B}} \mathcal{L}^{\circ n}(1) d \mathrm{~m}=\mathrm{m}\left(T^{-n}(\mathcal{B})\right) .
$$

On en déduit que

$$
\forall x \in \mathcal{B} \quad g(x) \sim \liminf _{n \rightarrow \infty} \mathrm{m}\left(T^{-n}(\mathcal{B})\right) .
$$

Or, cette limite inférieure est nécessairement strictement positive. Si tel n'était pas le cas, du théorème de Lebesgue on tirerait que la limite inférieure de la suite $\left(\mu\left(T^{-n}(\mathcal{B})\right)\right)_{n \in \mathbb{N}}$ est elle aussi nulle. Or, cette suite est stationnaire puisque $\mu$ est $T$-invariante. On aurait donc $\mu(\mathcal{B})=0$. C'est impossible puisque $\mathrm{m}(\mathcal{B})>0$ et $\mathrm{m}$ et $\mu$ sont équivalentes. La fonction $g$ n'est donc pas identiquement nulle sur $J$ et $g \mathrm{~m}$ est bien, à une constante multiplicative près, la probabilité $T$-invariante $\mu$.

REMARQUe. Ce raisonnement appliqué dans le cas $\alpha(T) \leq 2$ nous prouve que

$$
\forall x \in \widehat{\mathbb{C}} \backslash \mathcal{P C} \quad \liminf _{n \rightarrow \infty} \mathcal{L}^{\circ n}(1)(x)=0,
$$

et aussi que pour toute boule $\mathcal{B}$ éloignée de $\mathcal{P C}$ on a

$$
\liminf _{n \rightarrow \infty} \mathrm{m}\left(T^{-n}(\mathcal{B})\right)=0 .
$$

La deuxième égalité est une conséquence de la première et de (2.3).

La première égalité est obligatoirement vérifiée m-presque partout. Sinon $g$ m est une mesure non nulle, finie, $T$-invariante et absolument continue par rapport à $\mathrm{m}$. Sous l'hypothèse $\alpha \leq 2$ une telle mesure ne peut pas exister. La première égalité est donc vraie m-presque partout. Pour conclure qu'elle est vraie dans $\widehat{\mathbb{C}} \backslash \mathcal{P C}$ il suffit d'appliquer (2.2).

Dans le lemme suivant, nous précisons le comportement de $g$.

Lemme 4.1. (a) g est une fonction continue sur $J \backslash \mathcal{P C}$. 
(b) Pour tout $k \geq 1$ et tout $\omega \in \mathcal{P C} \cap J$ on a

$$
\forall x \in \mathcal{M}_{\omega, k} \quad g(x) \sim \frac{\mu\left(\mathcal{M}_{\omega, k}\right)}{\mathrm{m}\left(\mathcal{M}_{\omega, k}\right)} .
$$

En utilisant les propositions 2.1 et 3.2 on obtient à l'aide de ce lemme une estimation assez précise de la grandeur de $g$ sur $\mathcal{M}_{\omega, k}$ en fonction de $k$ et de la nature de $\omega$. On en déduit aussi le

Corollaire 4.2. Pour tout $x$ élément de $J \backslash \mathcal{P C}$ on a $\mathcal{L}(g)(x)=g(x)$.

Démonstration. On a vu que $\mathcal{L}(g)=g$ m-presque sûrement. Or, $\mathrm{m}$ est une mesure borélienne qui charge les ouverts de $J$. Comme $g$ est continue sur $J \backslash \mathcal{P C}$ l'égalité presque sûrement s'étend en une égalité en tout point de $J \backslash \mathcal{P C}$.

Démonstration du lemme 4.1. (a) Soit $x_{0}$ un point de $J \backslash \mathcal{P C}$. On prend une boule $\mathcal{B}=B\left(x_{0}, r\right)$ centrée en $x_{0}$ et suffisamment éloignée de $\mathcal{P C}$ pour que la boule ayant un rayon double soit elle aussi éloignée de $\mathcal{P C}$. Ainsi, sur $\mathcal{B}^{\prime}=B\left(x_{0}, 2 r\right)$ toutes les branches holomorphes de $T^{-n}$ sont holomorphes et injectives. La proposition 2.2 nous indique alors que pour toute composante connexe $\mathcal{B}_{n}$ de $T^{-n}(\mathcal{B})$ on a

$$
\forall(x, y) \in \mathcal{B}_{n} \times \mathcal{B}_{n} \quad e^{-\lambda \varepsilon(|x-y|)} \leq \frac{\left|\left(T^{\circ n}\right)^{\prime}(x)\right|}{\left|\left(T^{\circ n}\right)^{\prime}(y)\right|} \leq e^{\lambda \varepsilon(|x-y|)} .
$$

Comme $\lambda$ et $\varepsilon$ sont indépendants de $n$ et de la composante connexe de $T^{-n}(\mathcal{B})$ considérée, on en déduit que

$$
\forall x \in \mathcal{B} \quad e^{-\lambda \varepsilon\left(\left|x-x_{0}\right|\right)} \mathcal{L}^{\circ n}(1)(x) \leq \mathcal{L}^{\circ n}(1)\left(x_{0}\right) \leq e^{\lambda \varepsilon\left(\left|x-x_{0}\right|\right)} \mathcal{L}^{\circ n}(1)(x) .
$$

On en déduit, par passage à la limite inférieure, que

$$
\forall x \in \mathcal{B} \quad e^{-\lambda \varepsilon\left(\left|x-x_{0}\right|\right)} g(x) \leq g\left(x_{0}\right) \leq e^{\lambda \varepsilon\left(\left|x-x_{0}\right|\right)} g(x) .
$$

Ces inégalités nous donnent la continuité de $g$.

(b) Pour ces estimations il suffit de prouver l'existence d'une constante $\lambda>0$ telle que pour tout $k$ et tout $\omega$ on ait

$$
\forall x \in \mathcal{M}_{\omega, k} \quad \frac{1}{\lambda} g(x) \leq g(y) \leq \lambda g(x) .
$$

Comme $\mu=g$ m est la mesure $T$-invariante, on a alors, pour tout $x$ dans $\mathcal{M}_{\omega, k}$

$$
\mu\left(\mathcal{M}_{\omega, k}\right)=\int_{\mathcal{M}_{\omega, k}} g d \mathrm{~m} \sim g(x) \mathrm{m}\left(\mathcal{M}_{\omega, k}\right) .
$$

C'est l'estimation recherchée.

Comme nous l'avons vu lors de la démonstration de la proposition 2.1, si $\omega$ est un point de $\mathcal{P C} \cap J$ alors

$$
\mathcal{M}_{\omega, k+1}=f_{\omega}^{-k}\left(\mathcal{M}_{\omega} \backslash f_{\omega}^{-1}\left(\mathcal{M}_{\omega}\right) \cap J\right) .
$$


$f_{\omega}$ est égale à $T$ si $\omega$ est un point fixe et est égale à $g_{\omega}$ (cf. les rappels concernant la dynamique holomorphe) si $\omega$ est un point critique. On rappelle de plus que $f_{\omega}^{-1}$ est la réciproque de $f_{\omega}$ qui a $\omega$ pour point fixe.

On suppose que $\omega$ est un point fixe de $T$. Alors $\mathcal{M}_{\omega} \backslash f_{\omega}^{-1}\left(\mathcal{M}_{\omega}\right) \cap J$ est un ensemble éloigné de $\mathcal{P C}$. On peut par conséquent le recouvrir par un nombre fini de boules éloignées de $\mathcal{P C}$ et telles que $f_{\omega}^{-k}\left(B_{i}\right)$ soit pour tout $k$ inclus dans $\mathcal{M}_{\omega}$. On note $\left(B_{i}\right)_{1 \leq i \leq K}$ ces boules. Pour tout couple $(i, j)$, $i \neq j$, on peut construire, comme cela a été fait lors de la démonstration de la proposition 2.1, deux voisinages simplement connexes de $B_{i} \cup B_{j}, \mathcal{V}_{i, j} \subset \mathcal{V}_{i, j}^{\prime}$, sur lesquels soit applicable la proposition 2.2. Le (b) de la proposition 2.3 nous donne alors l'existence d'une constante $\lambda_{i, j}>1$ telle que pour toute composante connexe, $\mathcal{V}_{i, j}^{k}$, de $T^{-k}\left(\mathcal{V}_{i, j}\right)$ on ait

$$
\forall n \in \mathbb{N} \forall\left(x, x^{\prime}\right) \in \mathcal{V}_{i, j}^{k} \quad \frac{1}{\lambda_{i, j}} \leq \frac{\mathcal{L}^{\circ n}(1)(x)}{\mathcal{L}^{\circ n}(1)\left(x^{\prime}\right)} \leq \lambda_{i, j} .
$$

On en déduit, par passage à la limite inférieure, que

$$
\forall\left(x, x^{\prime}\right) \in \mathcal{V}_{i, j}^{k} \quad \frac{1}{\lambda_{i, j}} g(x) \leq g\left(x^{\prime}\right) \leq \lambda_{i, j} g(x) .
$$

En particulier, il existe une constante $\lambda_{i, j}$ telle que

$$
\forall\left(x, x^{\prime}\right) \in f_{\omega}^{-k}\left(B_{i} \cup B_{j}\right) \quad \frac{1}{\lambda_{i, j}} g(x) \leq g\left(x^{\prime}\right) \leq \lambda_{i, j} g(x) .
$$

En posant $\lambda:=\sup \lambda_{i, j}$ on a

$$
\forall\left(x, x^{\prime}\right) \in \mathcal{M}_{\omega, k+1} \quad \frac{1}{\lambda} g(x) \leq g\left(x^{\prime}\right) \leq \lambda g(x) .
$$

Si maintenant $\omega$ est un point critique, $T(\omega)$ est un point fixe et un raisonnement similaire permet de conclure.

4.2. Description de la transition de phase dans le cas parabolique. Nous allons donc démontrer le théorème 1.3. Il affirme que les seuls états d'équilibre sont les Dirac aux points paraboliques, ou bien la probabilité $T$-invariante équivalente à la mesure $\delta$-conforme, ou encore une combinaison linéaire de ces deux types de mesures.

On note $\Omega$ l'ensemble des points paraboliques.

Remarque. Comme $T$ est supposée parabolique, son ensemble de Julia ne contient pas de points critiques. Au vue du théorème 4 de [De, $\mathrm{Ur}^{1}$ ], l'action de $T$ sur son ensemble de Julia est expansive. Soit $\nu$ une probabilité borélienne, $T$-invariante. Cette expansivité nous assure que tout recouvrement fini de $J(T)$ par des boréliens de diamètre inférieur à une constante d'expansivité, forme un générateur d'entropie finie pour $\nu$ (cf. $\left.\left[\mathrm{Wa}^{2}\right]\right)$. Cela 
implique (cf. $[\mathrm{Pr}, \mathrm{Ur}])$ que la formule de Rokhlin est applicable. On a donc

$$
h_{\nu}(T)=\int_{J} \log \mathcal{J}_{\nu} d \nu
$$

$\mathcal{J}_{\nu}$ désigne le jacobien de $\nu$. Cette fonction peut être caractérisée par

$$
\nu(T(A))=\int_{A} \mathcal{J}_{\nu} d \nu
$$

pour tout borélien $A \subset J$ sur lequel $T$ est injective.

Soit $\nu$ un état d'équilibre sans atome dans $J \backslash \Omega$. C'est donc une probabilité $T$-invariante et

$$
\int_{J} \log \mathcal{J}_{\nu} d \nu-\delta \int_{J} \log \left|T^{\prime}\right| d \nu=0 .
$$

En tant que mesure $T$-invariante, $\nu$ vérifie les deux propriétés suivantes :

$$
\sum_{T(y)=x} \frac{1}{\mathcal{J}_{\nu}(y)}=1 \quad \nu \text {-presque partout, }
$$

et si $f$ est une fonction $\nu$-intégrable, on dispose de la formule de changement de variables suivante :

$$
\int_{J}\left(\sum_{T(y)=x} \frac{1}{\mathcal{J}_{\nu}(y)} f(y)\right) d \nu(x)=\int_{J} f d \nu .
$$

Comme $\nu$ est, par hypothèse, à support dans $J \backslash \Omega$, nous voulons montrer que $\nu=\mu=g \mathrm{~m}$. En fait nous allons montrer que $\nu / g$ est une mesure $\delta$-conforme. Par unicité d'une telle mesure on aura donc $\nu / g=\mathrm{m}$. Le fait que $1 / g$ ne s'annule pas sur $J \backslash \Omega$ permet de conclure que l'on a bien $\nu=g \mathrm{~m}$.

On définit une fonction $\psi$ comme suit :

$$
\forall x \in J \backslash \Omega \quad \psi(x)=-\delta \log \left|T^{\prime}(x)\right|+\log \frac{g(x)}{g \circ T(x)} .
$$

On vérifie sans peine que pour tout $x$ dans $J \backslash \Omega$, on a

$$
\sum_{T(y)=x} e^{\psi(y)}=1 .
$$

D'autre part, nous le prouverons ultérieurement, $\psi$ est $\nu$-intégrable et on a

$$
\int_{J} \psi d \nu \geq-\delta \int_{J} \log \left|T^{\prime}\right| d \nu
$$

Un calcul élémentaire et (4.11) permettent de vérifier que pour tout $x$ dans $J \backslash \Omega$ on a

$$
\sum_{T(y)=x} \frac{1}{\mathcal{J}_{\nu}(y)}\left(\log \mathcal{J}_{\nu}(y)+\psi(y)\right) \leq \log \left(\sum_{T(y)=x} e^{\psi(y)}\right)=0
$$


avec égalité si et seulement si pour tout $y$ tel que $T(y)=x$ on a $\log \mathcal{J}_{\nu}(y)=$ $-\psi(y)$.

En intégrant cette inégalité par rapport à $\nu$, ce qui est possible puisque $\psi$ est $\nu$-intégrable d'après (4.12), on obtient

$$
\int_{J}\left(\sum_{T(y)=x} \frac{1}{\mathcal{J}_{\nu}(y)}\left(\log \mathcal{J}_{\nu}(y)+\psi(y)\right)\right) d \nu(x) \leq 0,
$$

avec égalité si et seulement si pour $\nu$-presque tout $x, \log \mathcal{J}_{\nu}(y)=-\psi(y)$ où $y$ est un antécédent de $x$. La formule de changement de variables (4.10) nous donne alors

$$
\int_{J}\left(\log \mathcal{J}_{\nu}+\psi\right) d \nu \leq 0
$$

avec égalité si et seulement si $\log \mathcal{J}_{\nu}=-\psi$ pour $\nu$-presque pour tout $x$.

D'autre part, $\log \mathcal{J}_{\nu}$ et $\psi$ sont $\nu$-intégrables, donc

$$
\int_{J}\left(\log \mathcal{J}_{\nu}+\psi\right) d \nu=\int_{J} \log \mathcal{J}_{\nu} d \nu+\int_{J} \psi d \nu \geq \int_{J} \log \mathcal{J}_{\nu} d \nu-\delta \int_{J} \log \left|T^{\prime}\right| d \nu=0 .
$$

Par conséquent,

$$
\int_{J}\left(\log \mathcal{J}_{\nu}+\psi\right) d \nu=0
$$

et $\log \mathcal{J}_{\nu}=-\psi \nu$-presque partout. Cela peut encore s'écrire

$$
\frac{g}{g \circ T} \mathcal{J}_{\nu}=\left|T^{\prime}\right|^{\delta} \quad \nu \text {-presque partout. }
$$

Comme d'après le lemme $4.1,1 / g$ est une fonction bornée, $\nu / g$ est une mesure finie dont le jacobien est précisément $\frac{g}{g \circ T} \mathcal{J}_{\nu}$.

$\nu / g$ a donc pour jacobien $\left|T^{\prime}\right|^{\delta}$. Il s'agit donc bien de la mesure $\delta$ conforme $\mathrm{m}$. Ainsi, $\nu=g \mathrm{~m}$ et le théorème 1.3 est démontré.

Il reste à prouver (4.12); cela revient à prouver que $\log \frac{g}{g \circ T}$ est $\nu$-intégrable et d'intégrale positive.

Proposition 4.3. $\log \frac{g}{g \circ T}$ est un élément de $L^{1}(\nu)$ et son intégrale est positive.

Démonstration. On note $\varphi_{n}$ la fonction caractéristique de $\bigcup_{k=0}^{n} \mathcal{U}_{k}$ $\cap \mathcal{M}$ et on pose $g_{n}=\varphi_{n} g+\left(1-\varphi_{n}\right)$. D'après le lemme 4.1 on sait que $\log g_{n}$ est bien définie et bornée. C'est donc une fonction $\nu$-intégrable. La $T$-invariance de $\nu$ nous donne

$$
\int_{J} \log \frac{g_{n} \circ T}{g_{n}} d \nu=0 .
$$

On remarque par ailleurs que $\log \frac{g_{n} \circ T}{g_{n}}$ converge $\nu$-presque partout vers $\log \frac{g \circ T}{g}$. Or, le lemme 4.1 permet de montrer que $\log \frac{g_{n} \circ T}{g_{n}}$ est minorée 
indépendamment de $n$. Le théorème de Fatou nous indique alors que

$$
\int_{J} \log \frac{g \circ T}{g} d \nu \leq \liminf _{n \rightarrow \infty} \int_{J} \log \frac{g_{n} \circ T}{g_{n}} d \nu=0
$$

C'est exactement

$$
\int_{J} \log \frac{g}{g \circ T} d \nu \geq 0
$$

La démonstration est terminée.

\section{Références}

[Aa,De,Ur] J. Aaronson, M. Denker and M. Urbański, Ergodic theory for Markov fibred systems and parabolic rational maps, Trans. Amer. Math. Soc. 337 (1993), 495-548.

[Bo,Zi] O. Bodart et M. Zinsmeister, Quelques résultats sur la dimension de Hausdorff des ensembles de Julia des polynômes quadratiques, Fund. Math. 151 (1996), 121-137.

[Bow] R. Bowen, Equilibrium States and the Ergodic Theory of Anosov Diffeomorphisms, Lecture Notes in Math. 470, Springer, 1975.

[De, Ur $\left.{ }^{1}\right] \quad$ M. Denker and M. Urbański, Hausdorff and conformal measures on Julia sets with a rationally indifferent periodic point, J. London Math. Soc. 43 (1991), 107-118.

$\left[\mathrm{De}, \mathrm{Ur}^{2}\right]-,-$, Absolutely continuous invariant measures for expansive rational maps with rationally indifferent periodic points, Forum Math. 3 (1991), 561-579.

[Fo] S. Foguel, The Ergodic Theory of Markov Processes, Van Nostrand, 1969.

$\left[\mathrm{McMu}^{1}\right] \quad$ C. McMullen, Hausdorff dimension and conformal dynamics 2: Geometrically finite rational maps, preprint, 1997.

$\left[\mathrm{McMu}^{2}\right]-$, Hausdorff dimension and conformal dynamics 3: Computation of dimension, preprint, 1997.

[Mi] J. Milnor, Dynamics in One Complex Variable: Introductory Lectures, Stony Brook IMS preprint, 1990.

[Po] C. Pommerenke, Boundary Behaviour of Conformal Maps, Springer, 1992.

[Pr,Ur] F. Przytycki and M. Urbański, Fractals in the complex plane-ergodic theory methods, to appear.

$\left[\mathrm{Ru}^{1}\right] \quad$ D. Ruelle, Thermodynamic Formalism, Addison-Wesley, 1978.

$\left[\mathrm{Ru}^{2}\right] \quad-$, Repellers for real analytic maps, Ergodic Theory Dynam. Systems 2 (1982), 99-107.

[Sm] S. Smirnov, Spectral analysis of Julia sets, thesis, California Institute of Technology, 1996.

[Su] D. Sullivan, Conformal dynamical systems, in: Geometric Dynamics, Lecture Notes in Math. 1007, Springer, 1983, 725-752.

[Ur $\left.{ }^{1}\right] \quad$ M. Urbański, Rational functions with no recurrent critical points, Ergodic Theory Dynam. Systems 14 (1994), 391-414.

$\left[\mathrm{Ur}^{2}\right]-$, Geometry and ergodic theory of conformal non-recurrent dynamics, ibid. 17 (1997), 1449-1476. 
$\left[\mathrm{Wa}^{1}\right] \quad \mathrm{P} . \mathrm{W}$ alters, A variational principle for the pressure of continuous transformations, Amer. J. Math. 97 (1976), 937-971.

$\left[\mathrm{Wa}^{2}\right]$-, An Introduction to Ergodic Theory, Springer, 1982.

MAPMO-UMR 6628

Université d'Orléans

B.P. 6759

45067 Orléans Cedex 2, France

E-mail: havard@labomath.univ-orleans.fr

Received 20 April 1998;

in revised form 5 August 1998 and 23 February 1999 